\title{
Molecular insights into the classification of high-grade endometrial carcinoma
}

\author{
Yaser R. Hussein ${ }^{1}$ and Robert A. SOSLOw ${ }^{2}$ \\ ${ }^{1}$ Department of Pathology, Morristown Medical Center, Morristown, and ${ }^{2}$ Department of \\ Pathology, Memorial Sloan Kettering Cancer Center, New York, NY, United States
}

\begin{abstract}
Summary
Endometrial carcinoma, which is associated with a mortality rate of approximately $20 \%$, is the most common gynecological malignancy in the Western world. It is a heterogeneous disease, with multiple histotypes, each constituting a different disease entity. However, interobserver diagnostic agreement is suboptimal, particularly among the most lethal histotypes. Most recent data also indicate that histotype assignment is not independently associated with survival, while in contrast, clinicopathological risk stratification and genomic classification are significantly prognostic. Recent work has shown that there are four molecular subgroups of endometrioid carcinomas instead of the two types proposed by Bokhman in the 1970s. Carcinomas with polymerase E (POLE) exonuclease domain hotspot mutations are highly prognostically favourable; those with copy-number alterations and TP53 mutations are highly aggressive; and microsatellite unstable and 'copy-number low' endometrioid carcinomas are associated with intermediate prognoses. This review summarises the genetic foundations of the various histotypes of endometrial carcinoma and synthesises this information in the form of algorithms, or classifiers, that recapitulate genomic classification that is not only prognostic, but also potentially diagnostic and therapeutically predictive. A review of Lynch syndrome and Lynch-like syndrome is also provided.
\end{abstract}

Key words: Endometrial carcinoma; high-grade carcinoma; TCGA; molecular classification; POLE; microsatellite instability.

Received 31 August, accepted 5 September 2017

Available online 13 December 2017

\section{INTRODUCTION}

Endometrial carcinoma is the most common gynecological malignancy in the Western world and accounts for approximately 74,000 deaths per year worldwide. ${ }^{1,2}$ Although the majority of endometrial carcinomas are generally low grade and low stage, with favourable prognoses, the high-grade group accounts for a disproportionate number of endometrial cancer deaths. ${ }^{3,4}$

From the pathogenetic point of view, Bokhman proposed that endometrial cancer can be classified into two types (type I and II) based on epidemiological, clinical, and endocrine characteristics. ${ }^{5}$ Type I tumours are low-grade and oestrogenrelated endometrioid carcinomas, and are associated with obesity, endometrial hyperplasia, and favourable outcomes. Type II tumours are oestrogen-independent, of nonendometrioid histology (mainly serous and clear cell carcinoma), are seen in post-menopausal women, and are associated with atrophic endometrium, and poor outcomes. While this dualistic model is useful and provided the framework for many studies that contributed to our current understanding of endometrial carcinomas, including their molecular alterations, it is imperfect and difficult to apply in clinical practice due to the significant overlap between type I and II tumours and substantial heterogeneity within each type. Many highgrade endometrial carcinomas, particularly International Federation of Gynecology and Obstetrics (FIGO) grade 3 endometrioid carcinomas and clear cell carcinomas, cannot be placed into either group.

Histologically, endometrial carcinomas are classified into the following subtypes based on the 2014 World Health Organization (WHO) classification system of gynecologic tumours: endometrioid carcinoma (FIGO grade 1, 2, and 3), serous carcinoma, clear cell carcinoma, undifferentiated/ dedifferentiated carcinoma, carcinosarcoma (formerly malignant mixed Müllerian tumour), neuroendocrine tumours, and mixed type. ${ }^{6}$ The traditional stratification of endometrial carcinoma by patient age, tumour grade, and clinical stage has been important in assessing prognosis and guiding surgical treatment and subsequent use of adjuvant therapy. Early-stage endometrioid cancers are generally treated by surgery, with or without adjuvant brachytherapy. Advancedstage carcinomas are treated with upfront surgery followed by chemotherapy, with or without radiation therapy. Histotyping, however, only has a profound influence on therapy when the carcinoma is low stage, as patients with serous carcinomas and carcinosarcomas typically undergo chemotherapy almost regardless of stage. Some data suggest that histotype may not be independently associated with clinical outcomes, ${ }^{8-10}$ as some histotypes, particularly serous carcinomas and carcinosarcomas, are intrinsically high grade, occur in older patients, and tend to present at an advanced stage. Other histotypes are highly heterogeneous from a morphological and genomic perspective, complicating efforts to link endometrioid or clear cell histotypes to specific clinical outcomes.

High-grade endometrial carcinoma constitutes a biologically, morphologically, genetically, and clinically heterogeneous group of tumours. Histologically, high-grade endometrial carcinomas are diagnostically recognisable in prototypic examples on review of haematoxylin and eosin (H\&E) slides, with or without immunohistochemistry; 
however, as many as $30 \%$ of high-grade endometrial carcinomas are not prototypic. ${ }^{9}$ Several studies have shown suboptimal interobserver reproducibility in the pathological diagnosis of high-grade endometrial carcinoma, even with the use of immunohistochemistry, ${ }^{9-11}$ limiting the ability to develop individualised treatments and calling for the need to improve and refine the current classification schemes. Over the past decade, many studies have assessed genetic alterations in endometrial carcinoma associated with each histological type. The recent application of next-generation sequencing technologies has led to a rapid and substantial shift toward understanding the molecular alterations in endometrial carcinoma. In 2013, The Cancer Genome Atlas Research (TCGA) network performed a comprehensive genomic and transcriptomic analysis of endometrioid and serous carcinomas and classified them into four molecular subgroups, including polymerase E (POLE)-ultramutated, microsatellite unstable/hypermutated, copy-number low/microsatellite stable, and copy-number high (serous and serouslike) tumours. ${ }^{12}$ The TCGA genomic classification system provided significant prognostic and potential therapeutic information; POLE-ultramutated tumours were associated with the best disease-free survival (DFS), copy-number high tumours were associated with the worst DFS, and the other two groups were associated with intermediate DFS. Subsequently, several investigators developed more practical methods to identify distinct subgroups with a prognostic signature, consistent with the molecular subgroups identified by TCGA, ${ }^{13,14}$ and attempted to apply this construct to other high-grade endometrial carcinomas, such as clear cell carcinoma $^{15}$ and undifferentiated and dedifferentiated carcinomas. ${ }^{16}$

In this review, we discuss the various histological types of high-grade endometrial carcinoma and the molecular aberrations and immunophenotype of each type. We also discuss the TCGA molecular classification of endometrial carcinomas, including the characteristics of each subgroup. Finally, we highlight the role of integrating molecular pathology into the current pathological classification scheme.

\section{HISTOLOGICAL CLASSIFICATION OF HIGH- GRADE ENDOMETRIAL CARCINOMA, IMMUNOPHENOTYPE AND GENOTYPE}

\section{FIGO grade 3 endometrioid carcinoma}

FIGO grade 3 endometrioid carcinoma demonstrates solid, trabecular or nested growth and may resemble poorly differentiated, non-keratinising squamous cell carcinoma (Fig. 1). Endometrioid glandular differentiation is usually focally present with or without other features that support endometrioid differentiation, including squamous and mucinous differentiation. ${ }^{6,17}$ At the molecular level, PTEN (a tumour suppressor gene), PIK3CA, and ARIDIA (a chromatin remodelling gene) are mutated in $>60 \%$ of FIGO grade 3 endometrioid carcinomas. ${ }^{12,18,19}$ KRAS and CTNNB1 ( $\beta$ catenin) are mutated in $40 \%,{ }^{12,18-20}$ while high somatic copy-number alterations and abnormal DNA ploidy are seen in $24 \%$ and $37 \%$ of such tumours, respectively. ${ }^{12,21}$ Overall, approximately $20-30 \%$ of endometrioid carcinomas are microsatellite instability-high (MSI-H), ${ }^{12,18-20,22,23}$ in most cases due to $M L H 1$ promoter methylation. TP53 mutations have been reported in $20-30 \%$ of FIGO grade 3

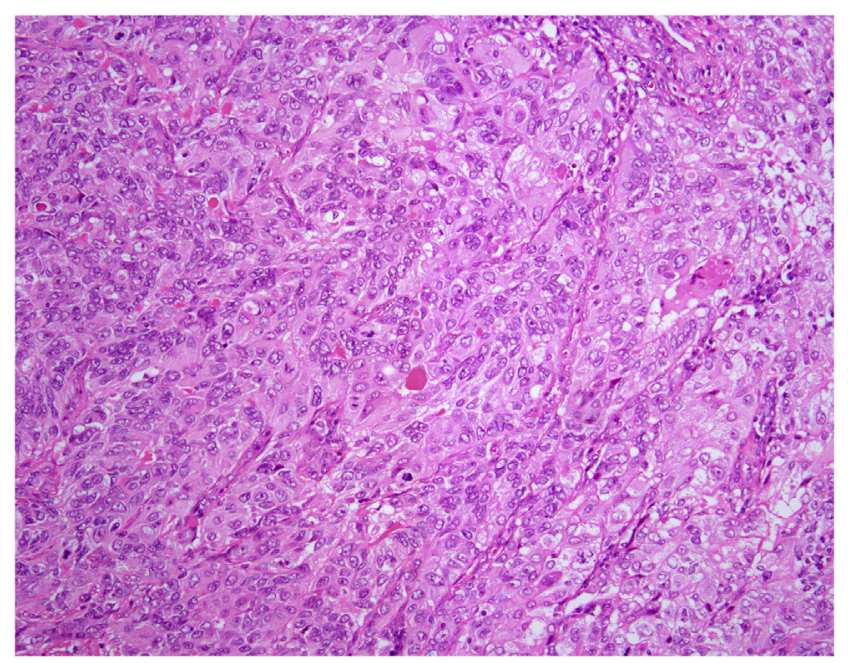

Fig. 1 International Federation of Gynecology and Obstetrics (FIGO) grade 3 endometrioid carcinoma with a solid growth pattern and nuclear atypia.

endometrioid carcinomas. ${ }^{12,19,24}$ Immunohistochemically, a large percentage of FIGO grade 3 endometrioid carcinomas show loss of PTEN, ARID1A (BAF250a), and/or DNA mismatch repair protein expression, and at least focal expression of oestrogen and progesterone receptors (ER and PR, respectively). ${ }^{25,26}$ However, PTEN staining can be difficult to interpret, as the antibody can be technically challenging to work with. ${ }^{26}$ Aberrant p53 and p16 expression is seen in a subset of FIGO grade 3 endometrioid carcinomas. $^{25}$ Aberrant p53 is usually in the form of overexpression ( $>60-75 \%$ strong uniform staining) corresponding to a missense mutation, or in a minority of cases, there will be complete loss of p53 staining in tumour cells in the presence of positive (wild-type positivity) internal control. This 'null-pattern' staining corresponds to a nonsense mutation, leading to the formation of a truncated protein that is not detected by commercially available p53 antibodies. $^{27,28}$ A recent report on TP53-mutated ovarian carcinomas described two additional very uncommon staining patterns that result from TP53 mutation: diffuse cytoplasmic staining and patchy, low-level expression that cannot be distinguished from the 'wild-type' staining pattern seen in tumours lacking TP53 mutation. ${ }^{29}$ To our knowledge, this has not yet been studied in the endometrium.

\section{Serous carcinoma}

Serous carcinoma typically shows irregular papillae with micropapillae and irregular luminal borders, slit-like spaces and tufted dyshesive cells exhibiting marked nuclear atypia and pleomorphism with smudgy chromatin or macronucleoli (Fig. 2). Occasionally, serous carcinoma shows predominantly glandular and/or solid architecture, which can mimic FIGO grade 3 endometrioid carcinoma. Cytoplasmic clearing might mimic clear cell carcinoma. Atrophic endometrium and/or an atrophic polyp is usually seen in the background. 6,17 At the genetic level, serous carcinoma is characterised by frequent mutations in TP53 (>90\%), PPP2R1A, $F B X W 7$, Her 2 amplification and high copy-number alterations, whereas mutations involving PTEN, ARIDIA, DNA mismatch repair genes, and KRAS are rare. ${ }^{12,18-20,30}$ Abnormal DNA ploidy is seen in the majority of serous 


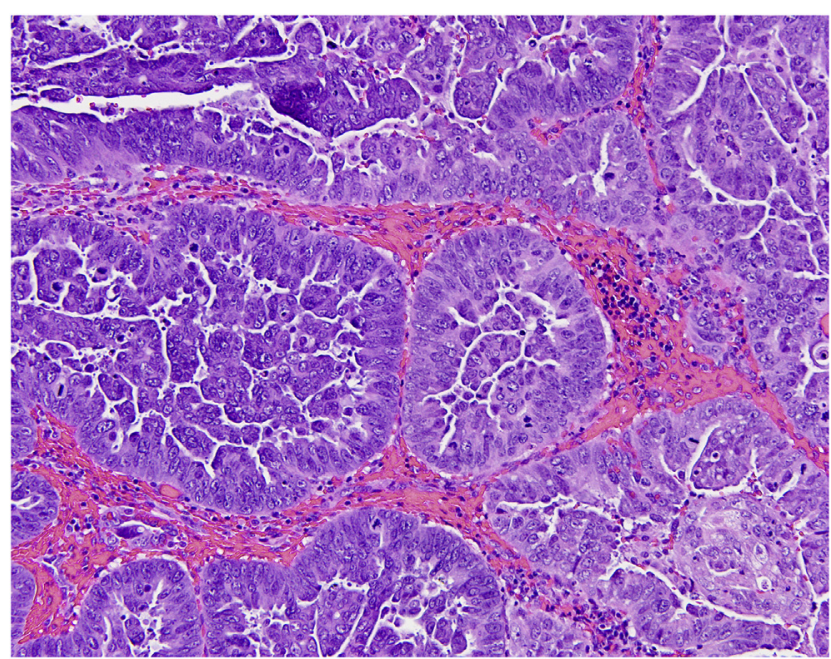

Fig. 2 Serous carcinoma exhibiting papillary and micropapillary patterns and diffusely atypical nuclei.

carcinomas. $^{23}$ In challenging cases, a panel of immunohistochemical stains, including p53, p16, PTEN, ARID1A and DNA mismatch repair proteins, may provide important ancillary information in the distinction between serous carcinoma and FIGO grade 3 endometrioid carcinoma. Typical serous carcinomas demonstrate aberrant p53 and p16 immunohistochemical stains, whereas ARID1A, PTEN and DNA mismatch repair proteins are usually retained. ${ }^{25,26,31-33}$ Up to $25 \%$ of serous and serous-like tumours show Her2 overexpression. $^{12}$

\section{Clear cell carcinoma}

Clear cell carcinoma is a rare type of endometrial carcinoma. It should be diagnosed predominantly based on cytoarchitectural features rather than cytoplasmic clearing, which can be occasionally seen in FIGO grade 3 endometrioid carcinoma or serous carcinoma. The papillae tend to be round with hyalinised cores, lined by one or two cell layers with uniform but high-grade nuclei (Fig. 3) ${ }^{6,17}$ TP53, PIK3CA, PPP2R1A, $F B X 7$, and ARIDIA are the most commonly mutated genes in endometrial clear cell carcinoma. ${ }^{15,34-36}$ Approximately $20 \%$

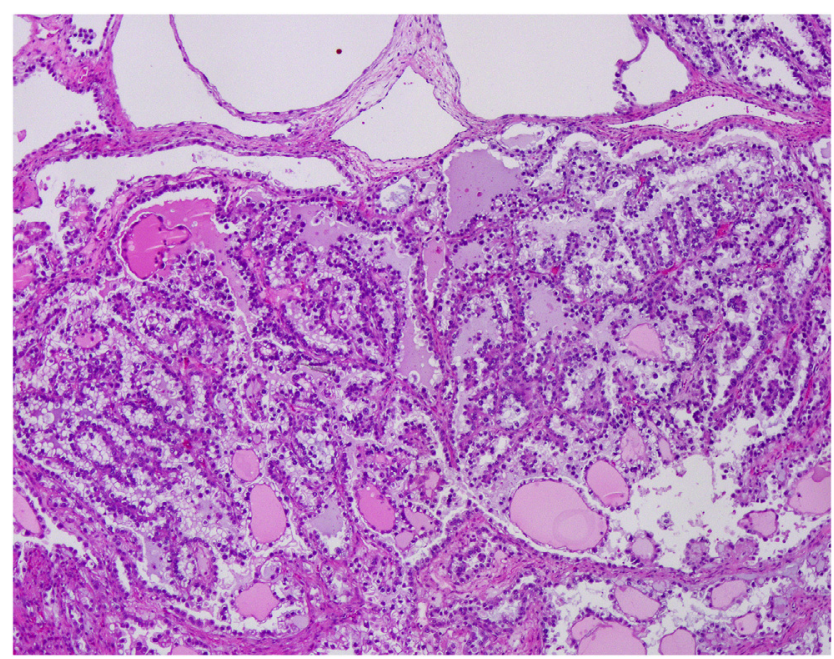

Fig. 3 Clear cell carcinoma with a predominantly papillary architecture. of endometrial clear cell carcinomas are MSI-H, and approximately $5 \%$ have a POLE mutation. ${ }^{15}$ The remaining endometrial clear cell carcinomas are TP53 mutated ( 35\%), have mutations usually associated with endometrioid carcinoma or a non-specific genotype. ${ }^{15}$ Immunophenotypically, clear cell carcinoma frequently shows expression of hepatocyte nuclear factor-1 $\beta$, Napsin-A and racemase, while ARID1A, ER and PR are frequently lost. ${ }^{33} \mathrm{~A}$ substantial minority shows overexpression of p53 and p16. ${ }^{26,35}$

\section{Undifferentiated/dedifferentiated carcinoma}

Undifferentiated and dedifferentiated endometrial carcinomas are part of an uncommon but highly aggressive family of tumours related to endometrioid carcinomas, ${ }^{37}$ and were first described by Silva and colleagues. ${ }^{38}$ Undifferentiated carcinoma, often mistaken for FIGO grade 3 endometrioid carcinoma, high-grade endometrial stromal sarcoma or lymphoma, is characterised by the presence of sheets of monotonous, ovoid to round cells with moderate-to-large nuclei, without convincing histological evidence of epithelial differentiation, such as glands, nests, trabeculae, or papillae (Fig. 4). Dedifferentiated carcinomas show foci of differentiated endometrioid carcinoma, usually low grade, juxtaposed against undifferentiated carcinoma without evident transitions. ${ }^{39}$ Undifferentiated carcinoma lacks diffuse expression of epithelial markers, such as pancytokeratin and E-cadherin, and it is frequently microsatellite unstable. ${ }^{37}$ Except for the very rare TP53-mutated examples, the tumours are negative for PAX8, ER, and PR. ${ }^{40}$ Recently, it has been shown that these carcinomas also show frequent loss of ARID1A, SMARCB1, and SMARCA4 by immunohistochemistry, suggesting a role for the SWI/SNF chromatin remodelling complex in the pathogenesis of this aggressive tumour. ${ }^{41,42}$ Rosa-Rosa et al. recently showed that undifferentiated endometrial carcinomas are genetically heterogeneous and may share molecular alterations with the four molecular subgroups of endometrial carcinoma described by TCGA, akin to FIGO grade 3 endometrioid carcinoma. ${ }^{16}$

Undifferentiated carcinomas are part of a family of carcinomas, including carcinosarcomas, thought to arise via epithelial-to-mesenchymal transition (EMT). EMT is a process by which epithelial cells lose polarity and intercellular adhesion, while acquiring properties of mesenchymal cells. EMT in uterine tumours typically involves upregulation of the miRNA 200 series, with consequent upregulation of established EMT-related proteins such as ZEB-1, SNAIL and TWIST, and downregulation of E-cadherin. ${ }^{43}$

\section{Carcinosarcoma}

Uterine carcinosarcomas are biphasic neoplasms composed of morphologically malignant epithelial and stromal elements (Fig. 5). The epithelial component is typically serous, FIGO grade 3 endometrioid carcinoma, or too poorly differentiated to subclassify. The stromal component is histologically high grade, featuring obvious nuclear atypia, pleomorphism, and mitotic activity. ${ }^{6,17}$ Heterologous elements, most commonly rhabdomyoblastic and chondrosarcomatous, are found in approximately half of cases. The sarcomatous component is thought to arise from metaplastic conversion due to strong EMT involving upregulation of the miRNA 200 series, as in undifferentiated and dedifferentiated carcinomas. ${ }^{43}$ From the molecular point of view, a subset of carsinosarcomas ( 20\%) 

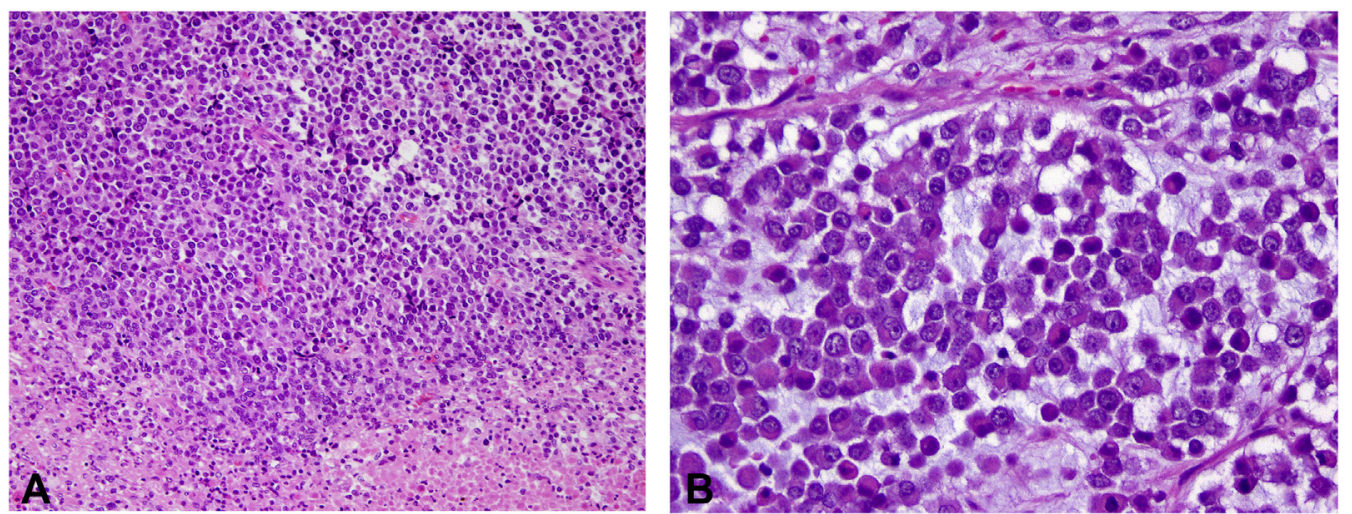

Fig. 4 Undifferentiated carcinoma. (A) Typical appearance: sheets of monomorphous tumour cells lacking any evident epithelial differentiation. (B) Undifferentiated carcinoma composed of rhabdoid cells floating in myxoid stroma.

shows mutations in PTEN, KRAS, ARIDIA, DNA mismatch repair genes, and $P O L E$ (i.e., endometrioid-like), while the majority share common molecular aberrations with ovarian and endometrial serous carcinoma, such as TP53 and PPP2R1A mutations, suggesting that while some carcinosarcomas arise from an endometrioid lineage, the majority transdifferentiate from a serous precursor. ${ }^{19,44}$,

\section{Mixed epithelial carcinoma}

Mixed carcinoma is a heterogeneous group of tumours composed of two or more distinct histological types (each must comprise at least $5 \%$ of tumour). ${ }^{6}$ Mixed carcinomas are difficult to classify and have poor interobserver reproducibility. ${ }^{45}$ Recent data reported by Köbel et al. showed shared clonality in both components of most mixed epithelial carcinomas studied. ${ }^{46}$ Sixteen of 18 mixed carcinomas contained elements with shared molecular alterations, despite showing different histological components. This suggests that most mixed carcinomas represent one tumour type with one component that morphologically mimics another type. $^{46,47}$ True mixed epithelial carcinomas are rare; the prototype is a microsatellite unstable mixed endometrioid and clear cell carcinoma. ${ }^{46}$

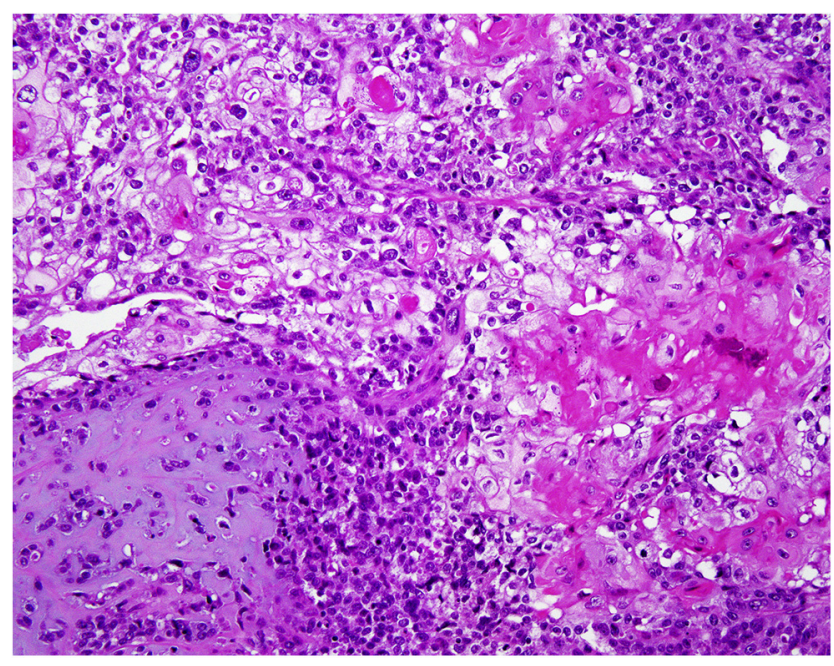

Fig. 5 Carcinosarcoma with a high-grade epithelial component (carcinoma, no otherwise specified, with squamous differentiation) and malignant chondroid component.

\section{TCGA GENOMIC CLASSIFICATION OF ENDOMETRIAL CARCINOMA}

In 2013, the multi-institutional TCGA project, sponsored by the National Cancer Institute and National Human Genome Research Institute, presented a molecular classification scheme for endometrial carcinoma. ${ }^{12}$ TCGA performed an integrated genomic, transcriptomic, and proteomic analysis of 373 endometrial carcinomas using massively parallel sequencing and array-based technologies in combination with DNA methylation, reverse phase protein array, and MSI analyses. The study focused on endometrioid $(n=307)$, serous $(n=53)$, and mixed endometrioid and serous $(n=13)$ carcinomas. Based on the integration of the somatic gene mutations, MSI and somatic copy-number alterations results, endometrial carcinomas were categorised into four genomic groups, as follows. ${ }^{12}$

1. POLE-ultramutated: An ultramutated group harbouring mutations in the exonuclease domain of POLE gene (a subunit of DNA polymerase epsilon that has a role in DNA replication) and characterised by very high mutation rates, few copy-number aberrations, increased frequency of $\mathrm{C} \rightarrow \mathrm{A}$ transversions, mutations in PTEN, PIK3CA, and $K R A S$, and highly favourable clinical outcomes. ${ }^{12}$

2. MSI-H/hypermutated: A hypermutated group of endometrial carcinomas, mostly of endometrioid histology, MSI-H due to $M L H 1$ promoter methylation, and characterised by high mutation rates, few copy-number alterations, and frequent KRAS and PTEN mutations. ${ }^{12}$ Lynch syndromeassociated endometrial carcinomas constitute a subset of the MSI-H tumours, which are discussed in detail below.

3. Copy-number low/microsatellite stable: A group of endometrioid carcinomas composed mostly of microsatellite-stable grade 1 and grade 2 tumours with low mutation rates, and characterised by frequent $P T E N$, CTNNB1, KRAS and ARIDIA mutations. ${ }^{12}$

4. Copy-number high (serous and serous-like): A group of tumours with extensive copy-number alterations (i.e., amplifications and deletions), low mutation rates and recurrent TP53, FBXW7, and PPP2RIA mutations, infrequent PTEN and $K R A S$ mutations, and composed of serous carcinomas and $25 \%$ of FIGO grade 3 endometrioid carcinomas. ${ }^{12}$

TCGA data provided a completely novel insight into the genetic and clinical heterogeneity of FIGO grade 3 
endometrioid carcinomas. Unlike serous carcinomas, FIGO grade 3 endometrioid carcinomas did not segregate into a single molecular cluster, but were represented in all four TCGA subgroups.

\section{TCGA GENOMIC CLASSIFICATION OF ENDOMETRIAL CARCINOMA, CLINICAL CORRELATIONS}

TCGA also conveyed crucial prognostic information. The genomic classification of endometrioid carcinomas stratified these tumours into clinically low-risk ( $P O L E$-ultramutated), intermediate-risk (copy-number low and MSI-H groups) and high-risk (copy-number high/serous-like) categories. With the exception of variable clinical outcomes based on clinical risk stratification, influenced heavily by stage, there is little apparent clinical heterogeneity within the POLE-ultramutated and copy-number high/serous-like groups. In contrast, there is obvious heterogeneity within the copynumber low group and some variability in the MSI-H group. In the copy-number low group, the presence of chromosome 1q amplification, a TP53 or CTNNB1 mutation predicts an increased risk for recurrence in a subset of lowgrade, low-stage endometrioid tumours. ${ }^{48}$ With regard to the MSI-H group, the clinical variability observed might be due to differences across centres with respect to staging adequacy and delivery of adjuvant therapy. In other words, withholding adjuvant therapy from patients with occult/undiscovered high-stage disease may be a particularly important negative prognostic factor in this group, as these tumours appear to be highly sensitive to adjuvant therapy. ${ }^{49-51}$ Finally, it is currently unclear whether there are important clinical differences between copy-number high (serous-like) endometrioid carcinomas and serous carcinomas.

For several years, there has been evidence showing that clear cell carcinomas with a TP53 mutation are prognostically unfavourable. $^{34,52}$ DeLair and colleagues recently published their comprehensive clinicopathological, immunophenotypic, and genomic evaluation of endometrial clear cell carcinomas. ${ }^{15}$ Even though four genomic clusters were recognised, similar to the TCGA results for endometrioid carcinomas, two clinical clusters emerged: prognostically highly favourable, represented by $P O L E$-ultramutated and hypermutated (MSI-H) carcinomas, and prognostically highly unfavourable, represented by TP53-mutated carcinomas and those lacking TP53 mutation, ultramutation, and hypermutation. ${ }^{15}$

Although both carcinosarcomas ${ }^{44}$ and un/de-differentiated carcinomas $^{53}$ contain POLE-ultramutated and MSI-H subgroups, there is currently insufficient information to determine their clinical relevance. As will be discussed below, it is possible that POLE-ultramutated and MSI-H tumours share unique features, irrespective of histotype, that may have prognostic and therapeutic relevance.

\section{ENDOMETRIAL CARCINOMAS WITH HIGH MUTATION BURDEN AND RICH IMMUNE MICROENVIRONMENT (POLE- ULTRAMUTATED AND MSI-H TUMOURS)}

These tumours have many unique biological, pathological, and clinical properties.

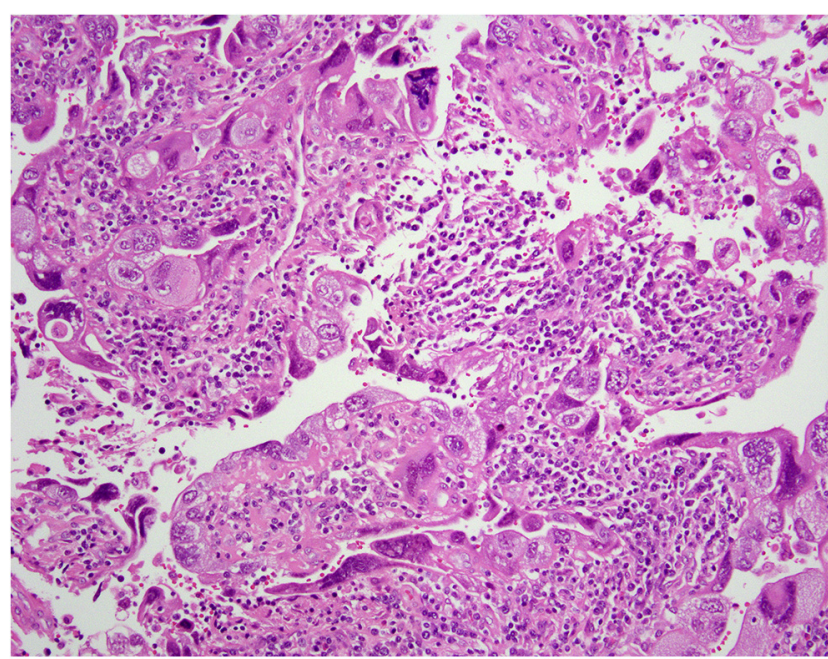

Fig. 6 POLE-mutated endometrioid carcinoma. POLE carcinomas may have eosinophilic tumour cells with giant, bizarre nuclei and a noticeable lymphoid infiltrate.

\section{POLE-ultramutated tumours}

POLE encodes the catalytic subunit of DNA polymerase epsilon. The resulting enzyme is involved in DNA repair and chromosomal DNA replication.

Detecting this subgroup of endometrial carcinomas was exciting, because of the discovery of very favourable clinical outcomes, even among high-grade tumours. Our group assessed the clinicopathological features of $P O L E$-ultramutated endometrial carcinomas in the TCGA series, as well as eight additional POLE-ultramutated tumours from an independent series of 99 high-grade endometrial carcinomas from the University of Calgary. ${ }^{54,55}$ The vast majority of POLE-ultramutated tumours presented at stage I. Most of them were FIGO grade 3 endometrioid carcinomas, rich in tumour-infiltrating lymphocytes and/or peritumoural (Crohn's-like) lymphocytes, and many of them showed marked intratumoural heterogeneity, with morphological ambiguity, including features mimicking serous carcinoma (Fig. 6). ${ }^{55}$ Approximately $8 \%$ of endometrial carcinomas overall and $15-20 \%$ of FIGO grade 3 endometrioid carcinomas show POLE exonuclease domain mutation and an ultratmutated genotype. ${ }^{12,56-59}$ Multiple studies have demonstrated that patients with POLE-ultramutated endometrial carcinoma have excellent prognoses, irrespective of other clinicopathological variables such as high histological grade and advanced stage. ${ }^{12,56-59}$ The reasons for such a favourable outcome are not completely understood; however, Meng et al. had previously speculated that this may be a result of the general or specific mutation burden conferred by the ultramutated genotype and the dramatic increase in base substitutions, which could be incompatible with tumour cell growth and survival. ${ }^{59} \mathrm{We}$ had previously suggested an immune-mediated role, given the rich tumour-infiltrating and peritumoural lymphocytes in these tumours. ${ }^{55}$ More recently, many researchers have investigated the role of tumourinfiltrating lymphocytes in POLE-ultramutated tumours. ${ }^{60-}$ ${ }^{62}$ Howitt et al. showed that POLE-ultramutated endometrial carcinoma is associated with high neoantigens and elevated CD8+ tumour infiltrating lymphocytes, which is counterbalanced by overexpression of program death-ligand 
1 (PD-L1; an immune checkpoint), suggesting that such tumours might be excellent candidates for anti-PD-L1 targeted therapies. ${ }^{60}$ Immune checkpoints other than PD-L1, such as cytotoxic T-lymphocyte antigen-4 (CTLA-4), lymphocyte activation gene-3 (LAG-3), and indoleamine 2,3dioxygenase (IDO), may also be upregulated in POLEultramutated tumours, which is known as 'adaptive immune resistance'. ${ }^{60,63-65}$ Although the discussion to this point was focused on somatic POLE hotspot mutations, germline mutations in POLE and polymerase D1 (POLDI) have also been reported to predispose to endometrial and colorectal carcinoma. ${ }^{56,66-68}$

\section{MSI-H endometrial carcinomas}

These tumours are typified by a microsatellite unstable phenotype that arises from defects in the post-replicative DNA mismatch repair system. MSI-H endometrial cancers are characterised by high mutation rates, few copy-number aberrations, recurrent KRAS and PTEN mutations, and few TP53, PPP2R1A, and FBXW7 mutations. Approximately $20-30 \%$ of endometrial carcinomas show MSI or mismatch repair protein abnormalities; ${ }^{12,18-21,23,64,69}$ this is most commonly due to sporadic events such as $M L H 1$ methylation.

MSI-H endometrial carcinomas are characterised by several morphological and topographical features (Fig. 7) including tumour-infiltrating lymphocytes, peritumoural lymphocytes, tumour heterogeneity, undifferentiated/dedifferentiated carcinoma, or non-endometrioid histology in young patients, particularly clear cell and mixed endometrioid and clear cell carcinomas, tumours originating in the lower uterine segment, and endometrial carcinoma associated with synchronous ovarian clear cell carcinoma. ${ }^{70-73}$ The prognostic impact of DNA mismatch repair status in endometrial carcinomas is unclear. Some studies have demonstrated that endometrial carcinomas associated with DNA mismatch repair deficiency and MSI are associated with poor prognostic indicators, including higher histological grade, presence of lymphovascular invasion and higher clinical stage, ${ }^{74,75}$ whereas others found no association with prognosis $^{76}$ or have shown improved clinical outcomes. ${ }^{22}$ As

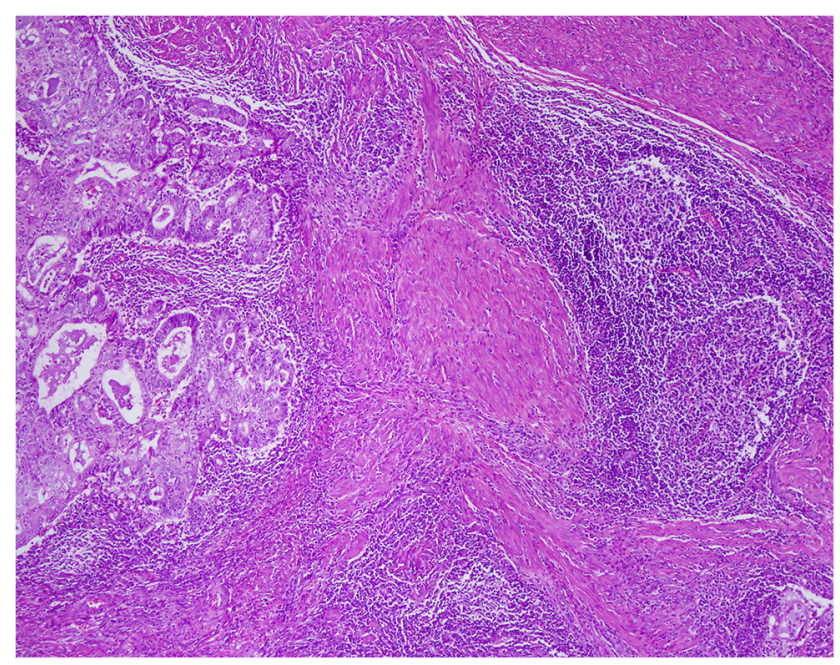

Fig. 7 Microsatellite-unstable endometrioid carcinoma showing a peritumoural Crohn's-like infiltrate and tumour infiltrating lymphocytes, a feature that is sometimes shared with POLE carcinomas. mentioned previously, the conflicting conclusions of various studies may reflect institutional differences in staging and delivery of adjuvant therapy. Similar to $P O L E$-ultramutated endometrial cancers, the presence of high mutation load, along with the rich immune microenvironment, make these tumours interesting potential candidates for immune checkpoint inhibitors such as anti-PD-L1 therapy. ${ }^{60,63-65}$

While most of the MSI-H tumours are sporadic, approximately $20-25 \%$ of MSI-H endometrial carcinomas are related to Lynch syndrome.

\section{Lynch syndrome}

This is an autosomal dominant cancer-susceptibility syndrome responsible for most heritable endometrial carcinomas and $<5 \%$ of ovarian carcinomas. Three general mechanisms, each of which impairs the DNA mismatch repair system, are responsible for the development of Lynch syndrome: (1) germline mutation in one of the DNA mismatch repair genes MLH1, MSH2, MSH6, or PMS2; (2) abnormalities in genes that regulate mismatch repair gene expression, such as EPCAM; or (3) constitutive epimutation. ${ }^{64,69}$ Germline mutation of one of the DNA mismatch repair genes is by far the most common and best understood mechanism underlying the development of Lynch syndrome. Germline EPCAM mutation, on the other hand, leads to $M S H 2$ promoter methylation, with consequent $M S H 2$ inactivation and acquisition of a MSI-H phenotype. Even though this scenario is considered part of the spectrum of Lynch syndrome, it has been reported that EPCAM-mutant Lynch syndrome families have more in common with Lynch-like syndrome (LLS) families (discussed below), meaning that the penetrance associated with the development of carcinoma may be muted with this genotype. ${ }^{77,78}$ Another rare mechanism underpinning the development of Lynch syndrome is the presence of intragenic insertions, deletions, and structural variations in the DNA mismatch repair genes, which are not always detectable using conventional assays. Next-generation deep DNA sequencing is more likely to identify these types of abnormalities when compared to Sanger DNA sequencing. ${ }^{79}$ Yet another rare example is DNA mosaicism in DNA derived from lymphocytes, usually used for germline DNA sequencing in Lynch syndrome diagnosis. ${ }^{99}$ This type of DNA mosaicism tends to be absent in the target organ, which in some cases harbours the pathogenic Lynch syndromedefining germline DNA mismatch repair mutations. All of these scenarios are difficult to identify and may explain clinical manifestations of Lynch syndrome when conventional germline gene mutation analysis appears negative (i.e., false negative). The use of next-generation sequencing has also uncovered novel DNA mismatch repair mutations, some of which have not been confirmed to be pathogenic. These are termed 'variants of uncertain (or unknown) significance' (VUS). Most of these cases previously would have appeared to be false-negative assays due to the inability of Sanger sequencing to detect the abnormality. Whether these cases constitute Lynch syndrome is uncertain.

Impairment of DNA mismatch repair leads to MSI and a predisposition for developing multiple malignancies, including colon and endometrial cancers, among others. ${ }^{64}$ While colon cancer has been the most commonly studied cancer in this disorder, women with Lynch syndrome have a significant lifetime risk of developing endometrial carcinoma 
and/or ovarian carcinoma that exceeds that of colon cancer, and in greater than $50 \%$ of cases, endometrial carcinoma is the first, or 'sentinel', malignancy. ${ }^{64,80,81}$ The identification of Lynch syndrome in endometrial carcinoma patients is important, as it provides the patient with the opportunity to be screened for synchronous and metachronous Lynch syndrome-associated tumours, particularly colon cancer. ${ }^{80}$ Furthermore, it allows their close family members to undergo screening for Lynch syndrome ${ }^{82}$ and then prophylactic hysterectomy and salpingo-oophorectomy, if appropriate. Germline DNA mismatch repair protein gene defects have been reported in 1.8-5.9\% of unselected patients with endometrial carcinoma. ${ }^{7,83-86}$ The optimal strategy for identifying patients with Lynch syndrome among newly diagnosed endometrial carcinoma patients, however, remains controversial. While age and family history are useful but rough screening criteria, their sensitivity has been shown to be significantly suboptimal in detection of Lynch syndromeassociated endometrial carcinoma. ${ }^{74,87}$ Testing tumours for DNA mismatch repair protein expression using immunohistochemistry with or without MSI assays has been shown to be a more sensitive and specific method for identifying patients. $^{74}$ In many institutions, these screening tests are performed on tumours from all patients diagnosed with endometrial carcinoma. Universal screening has been recently recommended by the American Society of Gynecologic Oncology, ${ }^{88}$ with the following rationale: (1) the incidence of Lynch syndrome-associated carcinomas increases with age and is by no means confined to younger patients; (2) Lynch syndrome screening identifies endometrial carcinoma patients at risk of developing another Lynch syndrome-associated carcinoma, such as colorectal carcinoma, which has medicolegal implications and, more importantly, profound clinical importance; and (3) Lynch syndrome screening of an index patient affords generations of Lynch syndrome family members the opportunity to be screened, allowing early detection of carcinoma and its prevention. Figure 8 shows an algorithm for the detection of endometrial carcinoma patients who are at high risk for having Lynch syndrome.

The importance of DNA mismatch repair immunohistochemistry is not only crucial for Lynch syndrome screening,

\section{Testing scheme}

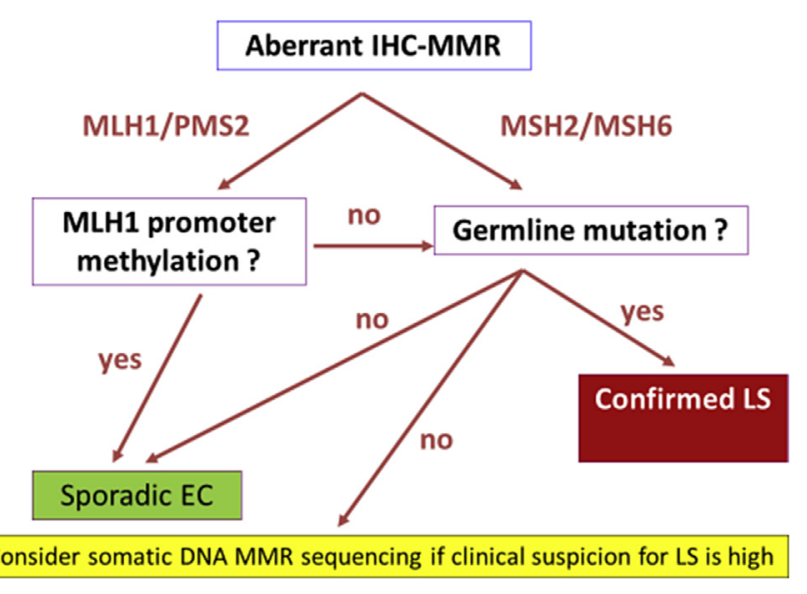

Fig. 8 Flow chart for Lynch syndrome triage using universal DNA mismatch repair immunohistochemistry as a starting point. but also for therapeutic prediction (i.e., sensitivity to immune checkpoint inhibitors, as discussed above), prognostication (i.e., assignment to a clinically relevant TCGA subgroup, as discussed above), and differential diagnosis, as discussed in following portions of this review.

\section{Lynch-like syndrome (LLS)}

LLS is diagnosed in the presence of an MSI-H and/or DNA mismatch repair-deficient carcinoma, lacking MLH1 promoter methylation, without an identifiable DNA mismatch repair germline mutation. Most of the work on this topic has been performed in the setting of colorectal carcinoma, and it is assumed that many of the underlying mechanisms that account for LLS in endometrial and ovarian carcinomas are likely similar, but other details need confirmation. LLS patients present at approximately the same age as Lynch syndrome patients (approximately 10-20 years younger than patients with sporadic carcinomas), have significantly lower rates of developing colorectal carcinoma, and less frequently have a family or personal history of Lynch syndromeassociated carcinomas. ${ }^{79,89-93}$ However, LLS patients and their first-degree relatives are thought to be at higher risk of developing colorectal carcinoma as compared to patients with sporadic endometrial carcinoma, irrespective of MSI status. LLS carcinomas constitute $30-60 \%$ of all MSI-H carcinomas lacking DNA mismatch repair germline mutation and MLH1 promoter methylation. The presence of rare, germline abnormalities, discussed previously, should be excluded before considering LLS.

The literature has emphasised the importance of bi-allelic somatic mutations that affect the DNA mismatch repair genes as a widespread mechanism for the development of an LLS-associated carcinoma. ${ }^{94-98}$ It has been postulated that the first hit might occur in a cancer precursor lesion, while the second hit occurs with the development of carcinoma. By virtue of the presence of somatic mutations and the absence of germline mutations, these patients lack a heritable cancer syndrome and, thus, it is not necessarily recommended that they undergo close surveillance to detect early colorectal carcinoma. A somatic mutation in polymerase E or polymerase D (POLE or POLD, respectively) with secondary somatic mutations in one or more of the DNA mismatch repair genes ${ }^{99}$ has been reported as an uncommon mechanism for the development of an LSS phenotype.

Lynch syndrome can be distinguished from LSS when well-understood abnormalities reported in the setting of LS can be documented. This typically requires nextgeneration sequencing. A somatic mutation of POLE, $P O L D$ and/or bi-allelic somatic mutation of one or more of the DNA mismatch repair genes effectively precludes a diagnosis of Lynch syndrome, meaning that these patients do not need the close surveillance required for Lynch syndrome patients. Furthermore, there is a spectrum of poorly understood abnormalities, such as the presence of a VUS, which may place the patient and her relatives at increased risk for the development of Lynch syndromeassociated carcinomas. Affected patients should consult with a clinical geneticist in collaboration with her treating physician to determine whether close clinical surveillance, such as that offered to known Lynch syndrome patients, is indicated. 
POLE

p.V411L (c.1231G>T)
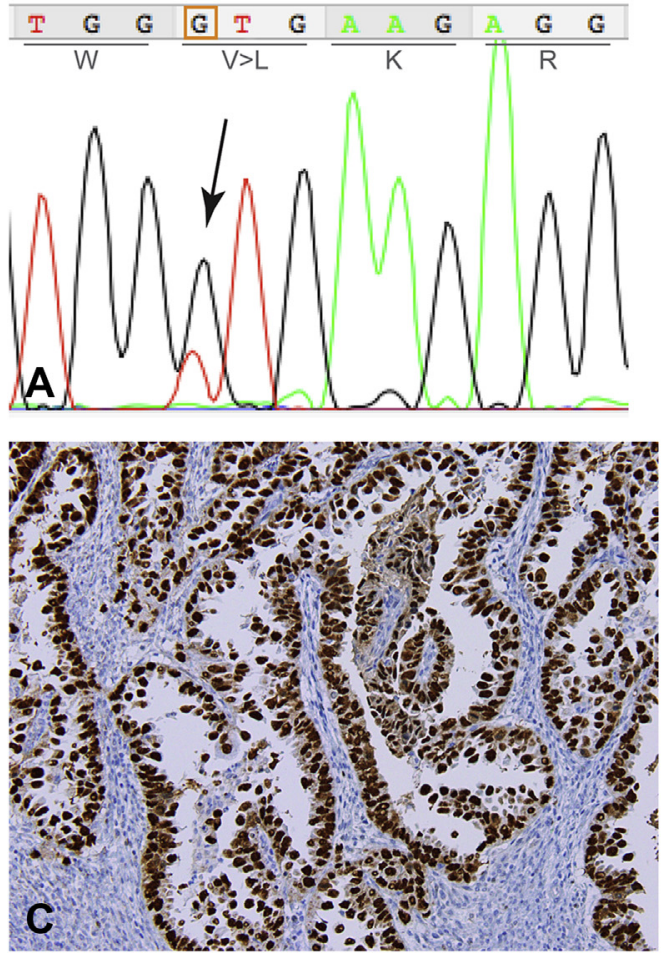
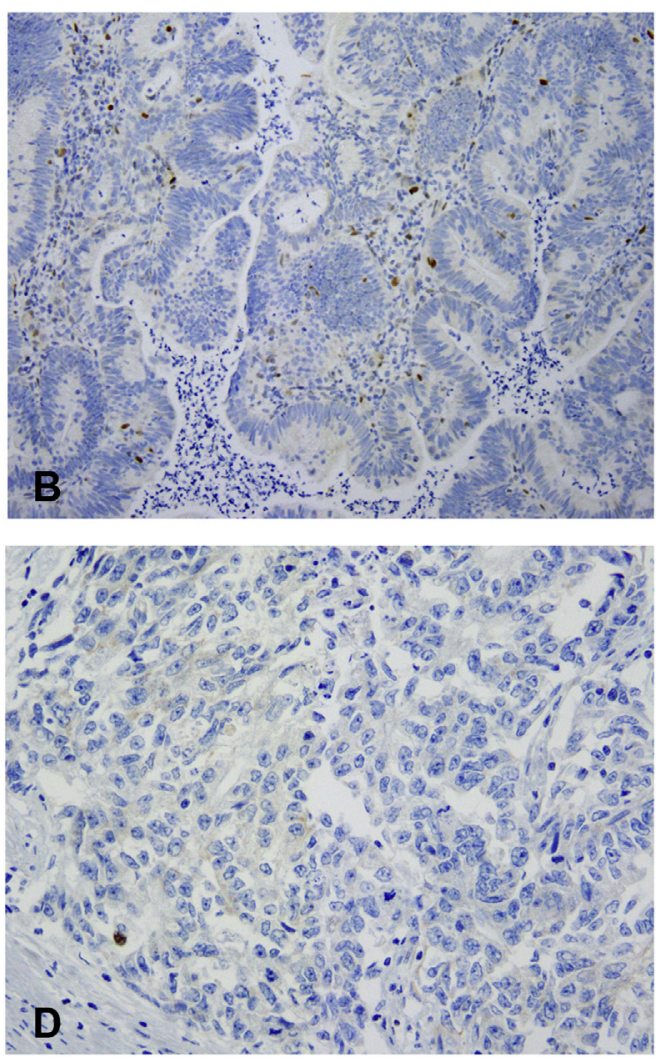

Fig. 9 (A-D) Endometrial carcinoma genotyping using POLE sequence and immunohistochemistry for the DNA mismatch repair proteins and p53. (A) POLE sequence (figure courtesy of Dr Britta Weigelt); (B) loss of expression of MSH6 in tumour cell nuclei, with an intact internal control; (C) aberrant p53 immunostaining (overexpression); (D) aberrant p53 immunostaining (no expression in tumour cell nuclei with an intact internal control).

\section{PRACTICAL APPLICATIONS OF TCGA DATA}

Many researchers have assessed the usefulness of incorporating next-generation sequencing into the differential diagnosis of high-grade endometrial carcinomas. ${ }^{12-14,61,88}$ This might be of particular interest in histologically ambiguous tumours that are difficult to classify and in tumours with non-informative immunohistochemical results. Several groups have proposed surrogate TCGA molecular classification systems. ${ }^{13,14,61}$ According to work from the Vancouver group, endometrioid and serous endometrial carcinomas can be assigned to one of the four TCGA groups without the extensive assays that originally generated the genomic clusters by combining POLE mutational analysis with p53 and DNA mismatch repair protein (MLH1, PMS2, MSH2 and MSH6) immunohistochemistry ${ }^{14}$ (Fig. 9) - the so-called 'PROMISE' classifier (Proactive Molecular Risk Classifier for Endometrial Cancer). An adaptation of the PROMISE classifier can be found in Fig. 10. Application of the classifier to 143 endometrial carcinomas resulted in the following distributions: POLE-ultramutated $9 \%$, microsatellite instable $32 \%$, copy-number low $18 \%$, and copynumber high (serous and serous-like) 39\%. In a multivariate analysis, both PROMISE classification and clinicopathologic risk group assignment [European Society for Medical Oncology (ESMO) guidelines] were significantly associated with clinical outcomes, whereas histotype was not. This model provides independent prognostic information beyond traditional clinicopathological variables and can be used in clinical practice. Other investigators have reported similar data. ${ }^{13}$
The integration of molecular testing has the potential to provide additional prognostic and predictive information to the traditional morphological, immunohistochemical, and clinical risk group assignment. Although the PROMISE classifier and related classifiers are feasible in resource-rich environments, there are obvious restrictions in other settings. That being said, there is little concern among pathologists and clinicians that resources be allocated to extensive testing of pulmonary and colorectal carcinomas, where targeted genetic profiling and immune checkpoint

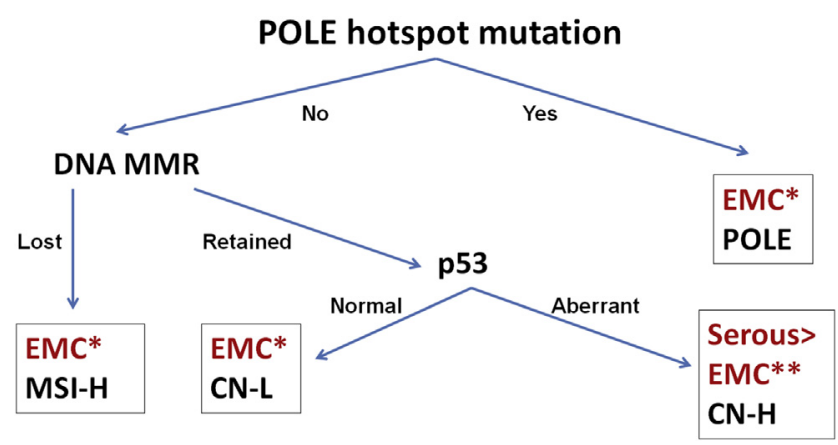

Fig. 10 Flow chart for endometrial carcinoma genotyping using available ancillary tests CN-H, copy number high (serous and serous-like); CN-L, copy number low; DNA MMR, DNA mismatch repair proteins; EMC, endometrioid carcinoma; MSI-H, microsatellite unstable; POLE, polymerase epsilon. *Applies mostly to endometrioid carcinoma, but also to undifferentiated and clear cell carcinomas. **Applies mostly to FIGO grade 3 endometrioid carcinoma, but also to clear cell carcinomas. Carcinosarcomas are found predominantly but not exclusively in the $\mathrm{CN}-\mathrm{H}$ group.. 
immunohistochemistry is standard in the former and DNA mismatch repair immunohistochemistry with frequent genetic profiling is performed in the latter. In the near future, there will be increasing interest, if not pressure, from educated clinicians for the molecular profiling of endometrial carcinoma as well. At present, this is best accomplished as outlined in the preceding text. Going forward, it is conceivable that both somatic and germline genetic testing of endometrial carcinomas and endometrial carcinoma patients will be performed using a multi-gene next-generation sequencing platform that will allow detection of somatic POLE and Tp53 mutations, as well as an MSI score. With patient consent, such technology will be able to identify germline POLE, POLD, ${ }^{56,66-68}$ and DNA mismatch repair mutations as well.

\section{CONCLUSION}

In this review, we have discussed the traditional pathological classification and the recent molecular classification of endometrial carcinoma. The integration of molecular features into the current pathological classification may provide additional prognostic and predictive information that can better define biologically and clinically relevant subsets of endometrial cancer.

Conflicts of interest and sources of funding: The authors state that there are no conflicts of interest to disclose.

Address for correspondence: Robert A Soslow, MD, Department of Pathology, Memorial Sloan Kettering Cancer Center, 1275 York Ave, New York, NY 10065, United States E-mail: soslowr@mskcc.org

\section{References}

1. Ferlay J, Shin HR, Bray F, et al. Estimates of worldwide burden of cancer in 2008: GLOBOCAN 2008. Int J Cancer 2010; 127: 2893-917.

2. Siegel RL, Miller KD, Jemal A. Cancer statistics, 2017. CA Cancer J Clin 2017; 67: 7-30.

3. Creasman WT, Kohler MF, Odicino F, et al. Prognosis of papillary serous, clear cell, and grade 3 stage I carcinoma of the endometrium. Gynecol Oncol 2004; 95: 593-6.

4. Halperin R, Zehavi S, Langer R, et al. Uterine papillary serous carcinoma (pure and mixed type) compared with moderately and poorly differentiated endometrioid carcinoma. A clinicopathologic study. Eur Gynaecol Oncol 2002; 23: 300-4.

5. Bokhman JV. Two pathogenetic types of endometrial carcinoma. Gynecol Oncol 1983; 15: 10-7.

6. Kurman RJ, Carcangiu ML, Herrington CS, et al. WHO Classification of Tumours of the Female Reproductive Organs. 4th ed. Lyon: IARC, 2014.

7. Salvesen HB, Haldorsen IS, Trovik J. Markers for individualised therapy in endometrial carcinoma. Lancet Oncol 2012; 13: e353-61.

8. Memorial Sloan Kettering Cancer Center (MSKCC). Endometrial Cancer Nomogram. New York: MSKCC, 2017. https://www.mskcc. org/nomograms/endometrial

9. Gilks CB, Oliva E, Soslow RA. Poor interobserver reproducibility in the diagnosis of high-grade endometrial carcinoma. Am J Surg Pathol 2013; 37: $874-81$.

10. Hoang LN, McConechy MK, Kobel M, et al. Histotype-genotype correlation in 36 high-grade endometrial carcinomas. Am J Surg Pathol 2013; 37: 1421-32.

11. Han G, Sidhu D, Duggan MA, et al. Reproducibility of histological cell type in high-grade endometrial carcinoma. Mod Pathol 2013; 26: 1594-604.

12. Kandoth $\mathrm{C}$, Schultz N, Cherniack AD, et al. Integrated genomic characterization of endometrial carcinoma. Nature 2013; 497: 67-73.

13. Stelloo E, Nout RA, Osse EM, et al. Improved risk assessment by integrating molecular and clinicopathological factors in early-stage endometrial cancer-combined analysis of the PORTEC cohorts. Clin Cancer Res 2016; 22: 4215-24.
14. Talhouk A, McConechy MK, Leung S, et al. A clinically applicable molecular-based classification for endometrial cancers. $\mathrm{Br} J$ Cancer 2015; 113: 299-310.

15. DeLair DF, Burke KA, Selenica P, et al. The genetic landscape of endometrial clear cell carcinomas. J Pathol 2017; 243: 230-41.

16. Rosa-Rosa JM, Leskela S, Cristobal-Lana E, et al. Molecular genetic heterogeneity in undifferentiated endometrial carcinomas. Mod Pathol 2016; 29: 1390-8.

17. Oliva E, Soslow RA. High-grade endometrial carcinomas. Surg Pathol Clin 2011; 4: 199-241.

18. Matias-Guiu X, Prat J. Molecular pathology of endometrial carcinoma. Histopathology 2013; 62: 111-23.

19. McConechy MK, Ding J, Cheang MC, et al. Use of mutation profiles to refine the classification of endometrial carcinomas. J Pathol 2012; 228: 20-30.

20. Samarnthai N, Hall K, Yeh IT. Molecular profiling of endometrial malignancies. Obstet Gynecol Int 2010; 2010: 162363.

21. Lax SF, Kendall B, Tashiro H, et al. The frequency of p53, K-ras mutations, and microsatellite instability differs in uterine endometrioid and serous carcinoma: evidence of distinct molecular genetic pathways. Cancer 2000; 88: 814-24.

22. Black D, Soslow RA, Levine DA, et al. Clinicopathologic significance of defective DNA mismatch repair in endometrial carcinoma. J Clin Oncol 2006; 24: 1745-53.

23. Proctor L, Pradhan M, Leung S, et al. Assessment of DNA ploidy in the ProMisE molecular subgroups of endometrial cancer. Gynecol Oncol 2017; 146: 596-602.

24. Garg K, Leitao Jr MM, Wynveen CA, et al. p53 overexpression in morphologically ambiguous endometrial carcinomas correlates with adverse clinical outcomes. Mod Pathol 2010; 23: 80-92.

25. Alkushi A, Kobel M, Kalloger SE, et al. High-grade endometrial carcinoma: serous and grade 3 endometrioid carcinomas have different immunophenotypes and outcomes. Int J Gynecol Pathol 2010; 29: $343-50$.

26. Chiang S, Soslow RA. Updates in diagnostic immunohistochemistry in endometrial carcinoma. Semin Diagn Pathol 2014; 31: 205-15.

27. Kobel M, Reuss A, du Bois A, et al. The biological and clinical value of p53 expression in pelvic high-grade serous carcinomas. J Pathol 2010; 222: $191-8$.

28. Kuhn E, Kurman RJ, Vang R, et al. TP53 mutations in serous tubal intraepithelial carcinoma and concurrent pelvic high-grade serous carcinoma-evidence supporting the clonal relationship of the two lesions. J Pathol 2012; 226: 421-6.

29. Lee S, Piskorz AM, Le Page C, et al. Calibration and optimization of p53, WT1, and Napsin A immunohistochemistry ancillary tests for histotyping of ovarian carcinoma: Canadian Immunohistochemistry Quality Control (CIQC) experience. Int J Gynecol Pathol 2016; 35: 209-21.

30. Shih Ie M, Panuganti PK, Kuo KT, et al. Somatic mutations of PPP2R1A in ovarian and uterine carcinomas. Am J Pathol 2011; 178: 1442-7.

31. Alkushi A, Clarke BA, Akbari M, et al. Identification of prognostically relevant and reproducible subsets of endometrial adenocarcinoma based on clustering analysis of immunostaining data. Mod Pathol 2007; 20: $1156-65$.

32. Alvarez T, Miller E, Duska L, et al. Molecular profile of grade 3 endometrioid endometrial carcinoma: is it a type I or type II endometrial carcinoma? Am J Surg Pathol 2012; 36: 753-61.

33. Chen W, Husain A, Nelson GS, et al. Immunohistochemical profiling of endometrial serous carcinoma. Int J Gynecol Pathol 2017; 36 $128-39$.

34. Fadare O, Gwin K, Desouki MM, et al. The clinicopathologic significance of p53 and BAF-250a (ARID1A) expression in clear cell carcinoma of the endometrium. Mod Pathol 2013; 26: 1101-10.

35. Lax SF, Pizer ES, Ronnett BM, et al Clear cell carcinoma of the endometrium is characterized by a distinctive profile of p53, Ki-67, estrogen, and progesterone receptor expression. Hum Pathol 1998; 29: $551-8$.

36. Wiegand KC, Lee AF, Al-Agha OM, et al. Loss of BAF250a (ARID1A) is frequent in high-grade endometrial carcinomas. J Pathol 2011; 224: 328-33.

37. Tafe LJ, Garo K, Chew I, et al. Endometrial and ovarian carcinomas with undifferentiated components: clinically aggressive and frequently underrecognized neoplasms. Mod Pathol 2010; 23: 781-9.

38. Altrabulsi B, Malpica A, Deavers MT, et al. Undifferentiated carcinoma of the endometrium. Am J Surg Pathol 2005; 29: 1316-21.

39. Silva EG, Deavers MT, Bodurka DC, et al. Association of low-grade endometrioid carcinoma of the uterus and ovary with undifferentiated carcinoma: a new type of dedifferentiated carcinoma? Int J Gynecol Pathol 2006; 25: 52-8. 
40. Ramalingam P, Masand RP, Euscher ED, et al. Undifferentiated carcinoma of the endometrium: an expanded immunohistochemical analysis including PAX-8 and basal-like carcinoma surrogate markers. Int $J$ Gynecol Pathol 2016; 35: 410-8.

41. Hoang LN, Lee YS, Karnezis AN, et al. Immunophenotypic features of dedifferentiated endometrial carcinoma - insights from BRG1/INI1deficient tumours. Histopathology 2016; 69: 560-9.

42. Karnezis AN, Hoang LN, Coatham M, et al. Loss of switch/sucrose nonfermenting complex protein expression is associated with dedifferentiation in endometrial carcinomas. Mod Pathol 2016; 29: 302-14.

43. Romero-Perez L, Lopez-Garcia MA, Diaz-Martin J, et al. ZEB1 overexpression associated with E-cadherin and microRNA-200 downregulation is characteristic of undifferentiated endometrial carcinoma Mod Pathol 2013; 26: 1514-24.

44. Cherniack AD, Shen H, Walter V, et al. Integrated molecular characterization of uterine carcinosarcoma. Cancer Cell 2017; 31: 411-23.

45. Hoang LN, Kinloch MA, Leo JM, et al. Interobserver agreement in endometrial carcinoma histotype diagnosis varies depending on The Cancer Genome Atlas (TCGA)-based molecular subgroup. Am J Surg Pathol 2017: 41: 245-52.

46. Kobel M, Meng B, Hoang LN, et al. Molecular analysis of mixed endometrial carcinomas shows clonality in most cases. Am J Surg Patho 2016; 40: 166-80.

47. Espinosa I, D'Angelo E, Palacios J, et al. Mixed and ambiguous endometrial carcinomas: a heterogenous group of tumors with different clinicopathologic and molecular genetic features. Am J Surg Pathol 2016; 40: 972-81.

48. Kurnit KC, Kim GN, Fellman BM, et al. CTNNB1 (beta-catenin) mutation identifies low grade, early stage endometrial cancer patients a increased risk of recurrence. Mod Pathol 2017; 30: 1032-41.

49. Bilbao C, Lara PC, Ramirez R, et al. Microsatellite instability predicts clinical outcome in radiation-treated endometrioid endometrial cancer. Int J Radiat Oncol Biol Phys 2010; 76: 9-13.

50. McMeekin DS, Tritchler DL, Cohn DE, et al. Clinicopathologic significance of mismatch repair defects in endometrial cancer: an NRG Oncology/Gynecologic Oncology Group Study. J Clin Oncol 2016; 34 3062-8.

51. Resnick KE, Frankel WL, Morrison CD, et al. Mismatch repair status and outcomes after adjuvant therapy in patients with surgically staged endometrial cancer. Gynecol Oncol 2010; 117: 234-8.

52. DeLair DF, Soslow R. Endometrial clear cell carcinoma with and without aberrant p53 expression: a study of 16 cases. Mod Pathol 2012; 25: $265 \mathrm{~A}$.

53. Espinosa I, Lee CH, D'Angelo E, et al. Undifferentiated and dedifferentiated endometrial carcinomas with POLE exonuclease domain mutations have a favorable prognosis. Am J Surg Pathol 2017; 41: 1121-8.

54. Bakhsh S, Kinloch MA, Hoang LN, et al. Histopathological features of endometrial carcinomas associated with POLE mutations: implications for decisions about adjuvant therapy. Histopathology 2016; 68: 916-24.

55. Hussein YR, Weigelt B, Levine DA, et al. Clinicopathological analysis of endometrial carcinomas harboring somatic POLE exonuclease domain mutations. Mod Pathol 2015; 28: 505-14.

56. Church DN, Briggs SE, Palles C, et al. DNA polymerase epsilon and delta exonuclease domain mutations in endometrial cancer. Hum Mol Genet 2013; 22: 2820-8.

57. Church DN, Stelloo E, Nout RA, et al. Prognostic significance of POLE proofreading mutations in endometrial cancer. J Natl Cancer Inst 2015 107: 402.

58. McConechy MK, Talhouk A, Leung S, et al. Endometrial carcinomas with POLE exonuclease domain mutations have a favorable prognosis. Clin Cancer Res 2016; 22: 2865-73.

59. Meng B, Hoang LN, McIntyre JB, et al. POLE exonuclease domain mutation predicts long progression-free survival in grade 3 endometrioid carcinoma of the endometrium. Gynecol Oncol 2014; 134: 15-9.

60. Howitt BE, Shukla SA, Sholl LM, et al. Association of polymerase emutated and microsatellite-instable endometrial cancers with neoantigen load, number of tumor-infiltrating lymphocytes, and expression of PD-1 and PD-L1. JAMA Oncol 2015; 1: 1319-23.

61. Piulats JM, Guerra E, Gil-Martin M, et al. Molecular approaches for classifying endometrial carcinoma. Gynecol Oncol 2017; 145: 200-7.

62. van Gool IC, Eggink FA, Freeman-Mills L, et al. POLE proofreading mutations elicit an antitumor immune response in endometrial cancer. Clin Cancer Res 2015; 21: 3347-55.

63. Brahmer JR, Tykodi SS, Chow LQ, et al. Safety and activity of anti-PDL1 antibody in patients with advanced cancer. N Engl J Med 2012; 366: $2455-65$.

64. Lynch HT, Lynch JF. Lynch syndrome: history and current status. Dis Markers 2004; 20: 181-98.

65. Topalian SL, Hodi FS, Brahmer JR, et al. Safety, activity, and immune correlates of anti-PD-1 antibody in cancer. N Engl J Med 2012; 366: $2443-54$.
66. Bellido F, Pineda M, Aiza G, et al. POLE and POLD1 mutations in 529 kindred with familial colorectal cancer and/or polyposis: review of reported cases and recommendations for genetic testing and surveillance. Genet Med 2016; 18: 325-32.

67. Church JM. Polymerase proofreading-associated polyposis: a new, dominantly inherited syndrome of hereditary colorectal cancer predisposition. Dis Colon Rectum 2014; 57: 396-7.

68. Palles C, Cazier JB, Howarth KM, et al. Germline mutations affecting the proofreading domains of POLE and POLD1 predispose to colorectal adenomas and carcinomas. Nat Genet 2013; 45: 136-44.

69. Lynch HT, Lynch JF, Snyder CL, et al. EPCAM deletions, Lynch syndrome, and cancer risk. Lancet Oncol 2011; 12: 5-6.

70. Broaddus RR, Lynch HT, Chen LM, et al. Pathologic features of endometrial carcinoma associated with HNPCC: a comparison with sporadic endometrial carcinoma. Cancer 2006; 106: 87-94.

71. Carcangiu ML, Radice P, Casalini P, et al. Lynch syndrome-related endometrial carcinomas show a high frequency of nonendometrioid types and of high FIGO grade endometrioid types. Int J Surg Pathol 2010; 18: 21-6.

72. Shia J, Black D, Hummer AJ, et al. Routinely assessed morphological features correlate with microsatellite instability status in endometrial cancer. Hum Pathol 2008; 39: 116-25.

73. Westin SN, Lacour RA, Urbauer DL, et al. Carcinoma of the lower uterine segment: a newly described association with Lynch syndrome. $J$ Clin Oncol 2008; 26: 5965-71.

74. Garg K, Leitao Jr MM, Kauff ND, et al. Selection of endometrial carcinomas for DNA mismatch repair protein immunohistochemistry using patient age and tumor morphology enhances detection of mismatch repair abnormalities. Am J Surg Pathol 2009; 33: 925-33.

75. Jung A. Microsatellite instability and therapeutic sensitivity. (German.) Verh Dtsch Ges Pathol 2007; 91: 112-8.

76. Zighelboim I, Goodfellow PJ, Gao F, et al. Microsatellite instability and epigenetic inactivation of MLH1 and outcome of patients with endometrial carcinomas of the endometrioid type. J Clin Oncol 2007; 25: 2042-8.

77. Kang SY, Park CK, Chang DK, et al. Lynch-like syndrome: characterization and comparison with EPCAM deletion carriers. Int $J$ Cancer 2015; 136: 1568-78.

78. Kuiper RP, Vissers LE, Venkatachalam R, et al. Recurrence and variability of germline EPCAM deletions in Lynch syndrome. Hum Mutat 2011; 32: 407-14.

79. Buchanan DD, Rosty C, Clendenning M, et al. Clinical problems of colorectal cancer and endometrial cancer cases with unknown cause of tumor mismatch repair deficiency (suspected Lynch syndrome). Appl Clin Genet 2014; 7: 183-93.

80. Lu KH, Dinh M, Kohlmann W, et al. Gynecologic cancer as a "sentinel cancer" for women with hereditary nonpolyposis colorectal cancer syndrome. Obstet Gynecol 2005; 105: 569-74.

81. Vasen HF, Wijnen JT, Menko FH, et al. Cancer risk in families with hereditary nonpolyposis colorectal cancer diagnosed by mutation analysis. Gastroenterology 1996; 110: 1020-7.

82. de Jong AE, Hendriks YM, Kleibeuker JH, et al. Decrease in mortality in Lynch syndrome families because of surveillance. Gastroenterology 2006; 130: 665-71.

83. Ferguson SE, Aronson M, Pollett A, et al. Performance characteristics of screening strategies for Lynch syndrome in unselected women with newly diagnosed endometrial cancer who have undergone universal germline mutation testing. Cancer 2014; 120: 3932-9.

84. Hampel H, Frankel W, Panescu J, et al. Screening for Lynch syndrome (hereditary nonpolyposis colorectal cancer) among endometrial cancer patients. Cancer Res 2006; 66: 7810-7.

85. Mills AM, Liou S, Ford JM, et al. Lynch syndrome screening should be considered for all patients with newly diagnosed endometrial cancer. Am J Surg Pathol 2014; 38: 1501-9.

86. Ollikainen M, Abdel-Rahman WM, Moisio AL, et al. Molecular analysis of familial endometrial carcinoma: a manifestation of hereditary nonpolyposis colorectal cancer or a separate syndrome? J Clin Oncol 2005; 23: 4609-16.

87. Ryan P, Mulligan AM, Aronson M, et al. Comparison of clinical schemas and morphologic features in predicting Lynch syndrome in mutation-positive patients with endometrial cancer encountered in the context of familial gastrointestinal cancer registries. Cancer 2012; 118 $681-8$.

88. Lancaster JM, Powell CB, Chen LM, et al. Society of Gynecologic Oncology statement on risk assessment for inherited gynecologic cancer predispositions. Gynecol Oncol 2015; 136: 3-7.

89. Carethers JM. Differentiating lynch-like from lynch syndrome. Gastroenterology 2014; 146: 602-4.

90. Hampel H, Bennett RL, Buchanan A, et al. A practice guideline from the American College of Medical Genetics and Genomics and the National Society of Genetic Counselors: referral indications for cancer predisposition assessment. Genet Med 2015; 17: 70-87. 
91. Mensenkamp AR, Vogelaar IP, van Zelst-Stams WA, et al. Somatic mutations in MLH1 and MSH2 are a frequent cause of mismatch-repair deficiency in Lynch syndrome-like tumors. Gastroenterology 2014; 146: 643-6.

92. Mills AM, Sloan EA, Thomas M, et al. Clinicopathologic comparison of Lynch syndrome-associated and "Lynch-like" endometrial carcinomas identified on universal screening using mismatch repair protein immunohistochemistry. Am J Surg Pathol 2016; 40: $155-65$.

93. Moline J, Mahdi H, Yang B, et al. Implementation of tumor testing for lynch syndrome in endometrial cancers at a large academic medical center. Gynecol Oncol 2013; 130: 121-6.

94. Hampel H, Frankel WL, Martin E, et al. Screening for the Lynch syndrome (hereditary nonpolyposis colorectal cancer). N Engl J Med 2005; 352: $1851-60$
95. Haraldsdottir S, Hampel H, Tomsic J, et al. Colon and endometria cancers with mismatch repair deficiency can arise from somatic, rather than germline, mutations. Gastroenterology 2014; 147: 1308-16.

96. Leenen $\mathrm{CH}$, van Lier MG, van Doorn HC, et al. Prospective evaluation of molecular screening for Lynch syndrome in patients with endometrial cancer $\leq 70$ years. Gynecol Oncol 2012; 125: 414-20.

97. Rodriguez-Soler M, Perez-Carbonell L, Guarinos C, et al. Risk of cance in cases of suspected lynch syndrome without germline mutation. Gastroenterology 2013; 144: 926-32.e1; quiz e13-4.

98. Win AK, Buchanan DD, Rosty C, et al. Role of tumour molecular and pathology features to estimate colorectal cancer risk for first-degree relatives. Gut 2015; 64: 101-10.

99. Billingsley CC, Cohn DE, Mutch DG, et al. Polymerase varepsilon (POLE) mutations in endometrial cancer: clinical outcomes and implications for Lynch syndrome testing. Cancer 2015; 121: 386-94. 